\title{
Role of FDG-PET/CT in Identification of Histological Upgrade of Ductal Carcinoma in Situ (DCIS) in Needle Biopsy
}

\author{
Na Young Jung (iD) ${ }^{1}$, Bong Joo Kang (iD ${ }^{2,{ }^{*}}$, Sung Hun $\mathrm{Kim}^{2}$, Ie Ryung Yoo ${ }^{2}$, Yeon Soo $\mathrm{Lim}^{1}$ and Won Jong \\ Yoo $^{1}$ \\ ${ }^{1}$ Department of Radiology, Bucheon St. Mary's Hospital, College of Medicine, the Catholic University of Korea, Seoul, South Korea \\ ${ }^{2}$ Department of Radiology, Seoul St. Mary's Hospital, College of Medicine, the Catholic University of Korea, Seoul, South Korea \\ "Corresponding author: Department of Radiology, Seoul St. Mary's Hospital, College of Medicine, the Catholic University of Korea, Seoul, South Korea. Email: \\ lionmain@catholic.ac.kr \\ Received 2021 February 16; Revised 2021 July 30; Accepted 2021 August 02.
}

\begin{abstract}
Background: Accurate preoperative detection of the invasive components of ductal carcinoma in situ (DCIS) is essential for an appropriate treatment. ${ }^{18} \mathrm{~F}$-fluorodeoxyglucose (FDG) positron emission tomography/computed tomography (PET/CT) scan, which can indicate the metabolic activity and aggressiveness of breast cancer, may be used as one of the predictors of the invasive components of DCIS in needle biopsy.

Objectives: To determine whether the FDG-PET/CT findings are associated with the histological upgrade of DCIS in biopsy.

Patients and Methods: In this retrospective cohort, we reviewed 165 cases of DCIS in 162 patients, who underwent preoperative FDGPET/CT examinations between April 2008 and September 2015. The clinicopathological characteristics and FDG-PET/CT findings of the patients were compared with respect to cancer invasion. The predictors of DCIS upgrade to invasive cancer were also examined. Moreover, the diagnostic performance of visual and semi-quantitative analyses of FDG-PET/CT in predicting invasion was compared. The semi-quantitative analyses were based on the maximum standardized uptake value $\left(\mathrm{SUV}_{\max }\right)$, divided by the cutoff point in a receiver operating characteristic (ROC) curve analysis.

Results: The final pathological findings indicated 119 cases of pure DCIS and 46 cases of DCIS with invasion. The optimal SUV max $_{2}$ threshold was 1.9 in the ROC curve analysis. Young age, high SUVmax, positivity in the visual analysis of FDG-PET/CT, and large pathological tumor size were significantly more frequent in the DCIS + invasion group. The significant predictors of DCIS histological upgrade were age $(\mathrm{P}=0.011)$, $\mathrm{SUV}_{\max }(\mathrm{P}<0.001)$, visual analysis of FDG-PET/CT $(\mathrm{P}=0.004)$, and pathological tumor size $(\mathrm{P}=0.003)$ in the univariate analysis. In the multivariate analysis, the $\mathrm{SUV}_{\max }$ (odds ratio $[\mathrm{OR}]=3.31, \mathrm{P}=0.003$ ) and tumor size $(\mathrm{OR}=1.20, \mathrm{P}=$ 0.022 ) were significant when the model included the $S U V_{\max }$, age, and size (model 1). On the other hand, age $(\mathrm{OR}=0.96, \mathrm{P}=0.032)$, visual analysis $(\mathrm{OR}=4.67, \mathrm{P}=0.006)$, and tumor size $(\mathrm{OR}=1.25, \mathrm{P}=0.005)$ were significant predictors when the model included visual analysis, age, and size (model 2). The sensitivity was significantly higher in the visual analysis, whereas the specificity, positive predictive value (PPV), and accuracy were significantly higher in the semi-quantitative analysis.
\end{abstract}

Conclusion: FDG-PET/CT is a potentially useful imaging tool to predict the upgrade of DCIS to invasive cancer.

Keywords: Breast Cancer, ${ }^{18}$ F-fluorodeoxyglucose (FDG)-Positron Emission Tomography/Computed Tomography

\section{Background}

Ductal carcinoma in situ (DCIS) is one of the precursor lesions for invasive breast cancer. The frequency of DCIS has been increasing due to the widespread use of mammography screening and technical improvements (1-3). However, a considerable number of patients who are confirmed as DCIS in preoperative biopsy ( 0 - 37\%) upgrade to invasive carcinoma, based on the final surgical pathological findings (4-6). Therefore, accurate diagnosis of the invasive components of DCIS is essential for appropriate surgical planning, including axillary lymph node (LN) staging.
Besides, overdiagnosis and overtreatment are emerging issues in DCIS patients diagnosed via breast cancer screening. Therefore, in patients diagnosed with DCIS through biopsy, prediction of invasive cancer seems essential for selecting breast cancer treatment options $(7,8)$.

Multiple studies have been carried out to define the clinicopathological characteristics associated with an upgrade to invasive cancer in patients preoperatively diagnosed with DCIS (5-7, 9-15). The possible predictors include the patient's age, palpability, tumor size, tumor grade, biopsy method, and some immunohistochemi- 
cal markers. However, there is no consensus regarding the prognostic biomarkers that can be used to predict DCIS upgrades. Previous studies have demonstrated an association between the maximum standardized uptake value $\left(\mathrm{SUV}_{\max }\right)$ measured with ${ }^{18} \mathrm{~F}$-fluorodeoxyglucose (FDG)-positron emission tomography/computed tomography (PET/CT) and prognostic factors, such as histological type and grade, immunohistochemical factors, and proliferation index (16-19). It seems that the sensitivity of FDG$\mathrm{PET} / \mathrm{CT}$ is lower for DCIS than invasive ductal carcinoma (IDC) $(1,20)$, thereby limiting its diagnostic application for primary DCIS lesions. Nonetheless, FDG-PET/CT may be a potential tool for predicting the invasive components of DCIS in biopsy.

\section{Objectives}

This study aimed to evaluate whether the $S_{U V} V_{\max }$ and visual analysis of FDG-PET/CT were associated with the histological upgrade of DCIS in needle biopsy.

\section{Patients and Methods}

\subsection{Patients}

This retrospective cohort was approved by the institutional review board; the requirement for obtaining informed consent was waived. By reviewing the medical database, a total of 204 patients with a preoperative diagnosis of DCIS in biopsy and preoperative FDG-PET/CT scan were identified between April 2008 and September 2015. The exclusion criteria were as follows: patients who had not been treated with definitive surgery $(n=13)$; patients who had recently undergone an excisional biopsy $(n=7)$; and patients with DCIS and IDC at different sites within the same breast in biopsy, which were not separately described in the final pathology $(n=3)$.

During data collection, patients with at least one of the immunohistochemical parameters missing were excluded $(n=19)$. Five patients had two separate DCIS lesions in their breasts (three bilateral and two unilateral lesions). The three bilateral lesions were analyzed separately, while only representative unilateral lesions were included. Finally, a total of 165 cases (162 patients) were investigated in this study. The mean interval from biopsy until FDG-PET/CT scan was 13.6 days (range: 5 - 52 days).

\subsection{FDG-PET/CT Protocol}

All patients underwent preoperative FDG-PET/CT examinations, using a dedicated breast PET/CT scanner (Biograph Duo or Biograph True Point, Siemens Medical Solutions, Knoxville, TN, USA). The patients were asked to fast for at least six hours before the examinations. After measuring the serum glucose levels to confirm euglycemia (blood glucose < $130 \mathrm{mg} / \mathrm{dL}$ ), $3.7 \sim 5.5 \mathrm{MBq} / \mathrm{kg}$ of ${ }^{18} \mathrm{~F}-\mathrm{FDG}$ was injected as a saline infusion.

After 60 minutes of post-injection bed rest, the patients underwent a PET scan. The acquisition time was 2 3 minutes per bed position. All patients were in a supine position with their arms raised during the PET/CT scan. A non-contrast CT scan was acquired from the orbitomeatal line to the upper thigh ( $80 \mathrm{mAs}, 130 \mathrm{kV}$, slice thickness of $5 \mathrm{~mm}$ and $50 \mathrm{mAs}, 120 \mathrm{kV}$, and slice thickness of $5 \mathrm{~mm}$, respectively), immediately followed by the corresponding PET scan of the same region. The CT data was used for attenuation correction, and images were reconstructed, based on the standard ordered-subset expectation maximization algorithm.

\subsection{FDG-PET/CT Scan Interpretation}

FDG-PET/CT scans were retrospectively interpreted by a nuclear medicine physician with 14 years of experience. All PET/CT scans were reviewed in a workstation using Syngo Fusion Package (Siemens Medical Solutions, Knoxville, TN, USA). Any lesion with FDG uptake greater than the background parenchymal uptake at the site of pathologically proven DCIS was defined as positive in the visual analysis. To find the exact location of the lesion, lesion-by-lesion correlations were examined via conventional imaging. For the semi-quantitative analysis, the $\mathrm{SUV}_{\max }$ of FDG was measured by placing the regions of interest (ROIs) around the primary tumor site with perceptible FDG uptake. If no FDG uptake was perceptible in the visual analysis, the ROI for the $S_{U V} V_{\max }$ was drawn at the location corresponding to the tumor site, based on the conventional imaging findings. If the FDG uptake was perceptible in an axillary LN, the case was regarded as positive for axillary LN metastasis.

\subsection{Histopathological Analysis}

Percutaneous biopsies were performed for suspicious lesions found in the previous mammography or US image in all patients before preoperative MRI and FDG-PET/CT scan for staging. For this purpose, a US-guided 14-gauge core needle biopsy (CNB) was carried out for 138 cases, and stereotactic vacuum-assisted biopsy (VAB) was performed for 27 cases, depending on the physician's choice (based on the radiologist's report).

All patients underwent surgical treatment for DCIS of the breasts, including breast-conserving surgery $(n=89)$ and mastectomy $(n=76)$. The surgical pathological results were used as the reference standard. There were no cases with positive surgical margins. The reviewed histopathological findings included the size of DCIS lesion, 
the presence of axillary LN metastasis, estrogen receptor (ER) and progesterone receptor (PR) status, human epidermal growth factor receptor type 2 (HER-2) status, Ki-67 index, and epidermal growth factor receptor (EGFR) status of the primary tumor, based on the pathological findings. Tumor size was measured as the maximum diameter of the surgically resected specimen. Immunohistochemistry was also used to evaluate the expression of molecular markers, including ER, PR, HER2, Ki-67, and EGFR.

\subsection{Statistical Analysis}

Continuous variables are expressed as mean \pm standard deviation (SD) and median with range, while categorical variables are expressed as number and percentage. To determine if continuous variables were normally distributed, Kolmogorov-Smirnov test was performed. Only the patient's age showed a normal distribution. Comparisons between continuous variables were performed using independent t-test (for patient's age) or Mann-Whitney U test (for $\mathrm{SUV}_{\max }$ and tumor size), depending on the normal distribution of data. Different groups were compared using $\chi^{2}$ test and Fisher's exact test regarding the biopsy method; tumor grade on biopsy; high and low $\mathrm{SUV}_{\text {max }}$ (divided by the cutoff point); visual analysis of primary tumor and axillary LNs in FDG-PET/CT; axillary LN metastasis in pathology; ER and PR status; HER2 expression; Ki-67 index; and EGFR.

Moreover, univariate and multivariate logistic regression analyses were performed to determine the effects of independent variables on cancer upgrade to invasion. The multivariate models were plotted separately for $S_{U} V_{\max }$ and PET cancer detection, along with factors showing $\mathrm{P}<$ 0.05 in the univariate analysis $\left(\mathrm{SUV}_{\max }+\right.$ age + tumor size in model 1 and PET cancer detection + age + tumor size in model 2). Besides, the performance of $S_{U} V_{\max }$ and visual detectability of invasive cancer were compared between the semi-quantitative analysis (divided by the cutoff point on the receiver operating characteristics [ROC] curve) and visual analysis by FDG PET/CT. All statistical analyses were performed in SAS version 9.3 (SAS Institute Inc., Cary, NC, USA), and a P-value $<0.05$ was considered statistically significant.

\section{Results}

In this study, all patients were female, with a mean age of $52.7 \pm 10.2$ years (range: 26 - 82 years). The final pathology confirmed pure DCIS in 119 cases (Figure 1), DCIS with microinvasion in 20 cases, and IDC in 26 cases (Figure 2 ). Cases of microinvasion were included in the DCIS + invasion group, with an upgrade rate of 27.9\% (46/165). Repre- sentative cases of pure DCIS and upgrade to invasive cancer are shown in Figure 1 and Figure 2, respectively.

The optimal SUV $\max$ threshold was 1.9, based on the ROC curve analysis. The comparison of clinicopathological characteristics and FDG-PET/CT scan, according to the final pathological classification, are shown in Table 1. Young age $(\mathrm{P}=0.008)$, high $\mathrm{SUV}_{\max }(\mathrm{P}<0.001)$, positivity in the visual analysis of FDG-PET/CT ( $\mathrm{P}=0.002)$, and large pathological tumor size $(\mathrm{P}<0.001)$ were significantly more frequent in the DCIS + invasion group as compared to the pure DCIS group.

The analysis of predictive factors for DCIS upgrade is shown in Table 2. An upgrade to invasive cancer was significantly correlated with age $(\mathrm{P}=0.011), \mathrm{SUV}_{\max }(\mathrm{P}<0.001)$, visual analysis of FDG-PET/CT $(\mathrm{P}=0.004)$, and pathological tumor size $(\mathrm{P}=0.003)$ in the univariate logistic regression analysis. In the multivariate analysis of $\mathrm{SUV}_{\max }$, age, and tumor size (model 1), the $S_{U} V_{\max }$ and tumor size were found to be significant predictors. In the visual analysis with age and tumor size (model 2), all variables were found to be significant predictors.

The area under the curve (AUC) was 0.72 (95\% confidence interval [CI]: $0.64-0.81)$ in the semi-quantitative analysis with $\mathrm{SUV}_{\max }$ and 0.62 (95\% CI: 0.56 - 0.68) in the visual analysis of ${ }^{18} \mathrm{~F}$-FDG PET/CT. The sensitivity, specificity, positive predictive value (PPV), negative predictive value (NPV), and accuracy of predicting DCIS upgrade to invasive cancer were $78 \%, 56 \%, 41 \%, 87 \%$, and $62 \%$ in the semiquantitative analysis with $\operatorname{SUV}_{\max }$ (cutoff point: 1.9) and $91 \%, 33 \%, 34 \%, 91 \%$, and $49 \%$ in the visual analysis of FDG PET/CT (Table 3). The sensitivity of visual analysis was significantly higher than that of the semi-quantitative analysis, whereas the specificity, PPV, and accuracy of the semiquantitative analysis were significantly higher than the visual analysis.

\section{Discussion}

FDG-PET/CT scan is not commonly recommended for DCIS patients with a low risk of metastasis. However, we hypothesized that this modality could be a useful preoperative diagnostic tool to predict the upgrade of DCIS to invasive cancer. The tumor cell number and nodular growth pattern were associated with FDG-PET/CT $(21,22)$. Therefore, the FDG uptake reflects not only the biological aggressiveness of a tumor but also the tumor cell density or tumor burden of DCIS, which is considered to be associated with invasion (22).

In this study, semi-quantitative anlysis based on $\mathrm{SUV}_{\text {max }}$ and visual analysis based on the reviwer's visual judgment were performed. In clinical practice, tumors with a low $\mathrm{SUV}_{\text {max }}$, such as DCIS, can be visually detected owing to the 

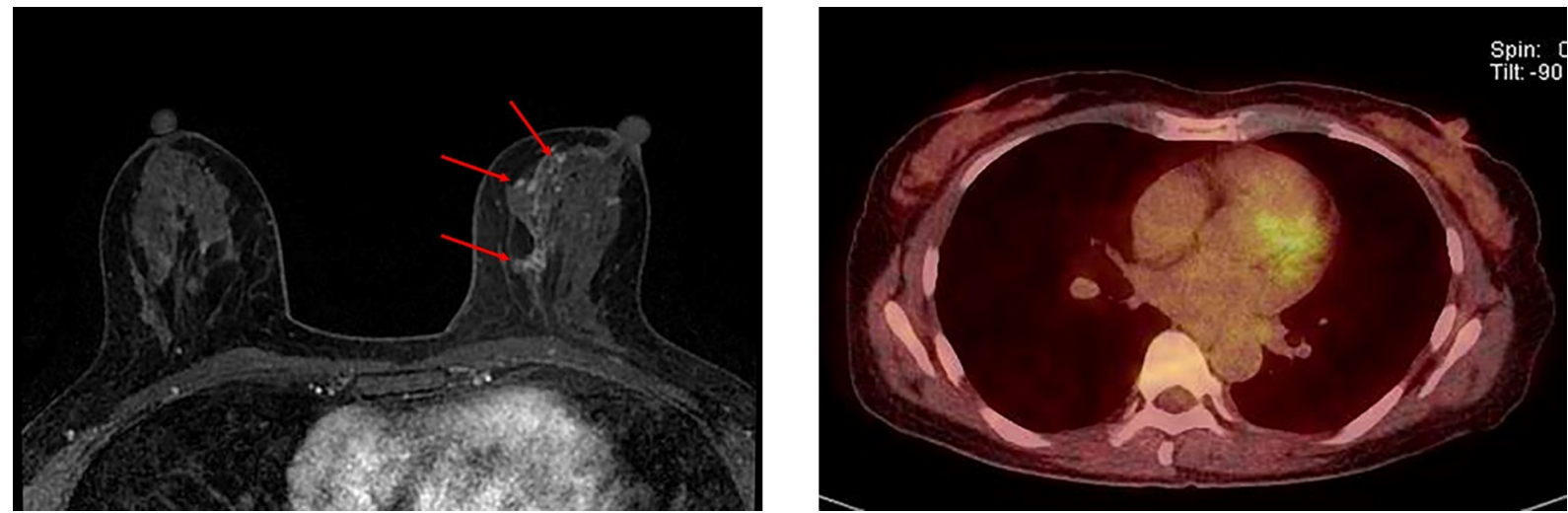

Figure 1. A 52-year-old woman with pure ductal carcinoma in situ (DCIS) in the left breast. A, Axial dynamic enhanced T1-weighted MRI demonstrates a segmental clumped non-mass enhancement $(3.5 \mathrm{~cm}$ ) (arrows) in the left middle inner part of the breast. B, The FDG-PET/CT scan shows no discernible FDG uptake in the left middle inner part of the breast.
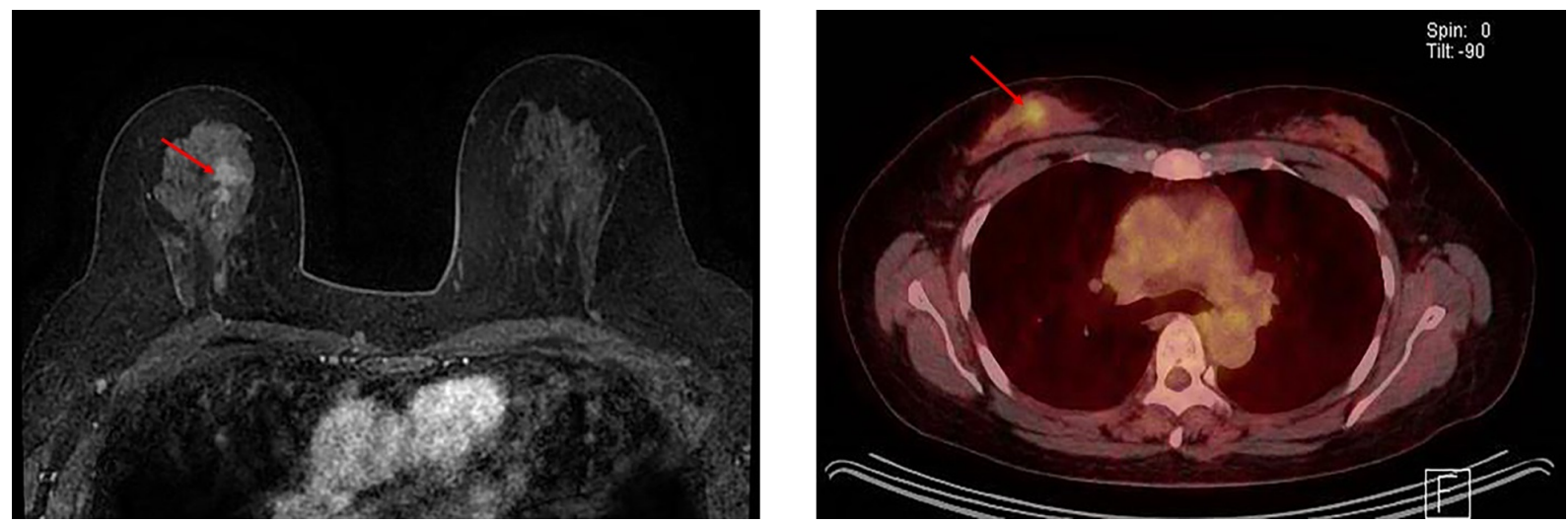

Figure 2. A 49-year-old woman with ductal carcinoma in situ (DCIS) upgraded to invasive ductal carcinoma (IDC) in the right breast. A, Axial dynamic enhanced T1-weighted MRI demonstrates a heterogeneously enhanced mass $(2.1 \mathrm{~cm})$ with an irregular shape and margins (arrow) in the right middle upper part of the breast. B, The FDG-PET/CT scan shows focal FDG uptake (arrow) in the right middle upper part of the breast with a maximum standardized uptake value (SUV $\max$ ) of 2.9

focal uptake pattern and relatively low background physiological absorption. Because of the low FDG uptake of DCIS, the semi-quantitative analysis has limited reliability; therefore, a more sensitive visual analysis was performed simultaneously. Some recent studies of DCIS upgrade, assessed by PET/CT scan, have conducted a visual analysis along with $\operatorname{SUV}_{\max }$ measurement $(22,23)$. In this study, the $S U V_{\max }$ and visual analysis were predictors of DCIS upgrade to invasive cancer in both univariate and multivariate models, similar to previous studies $(2,4)$.

Moreover, in previous research, the possible predictors of the invasive components of DCIS in biopsy were the patient's age $(7,11)$, palpability $(5,10)$, biopsy method $(5,6,11$, $13,14)$, tumor grade $(2,5,7,11,12,15)$, pathological or imaging tumor size $(5-7,9,11,13,15)$, and some immunohistochemical markers $(2,7,14,15)$. The patient's age was in- troduced as a predictor of DCIS upgrade in some previous studies $(7,11)$, but not others (22). In the present study, the patient's age was a significant predictor of DCIS upgrade to invasive cancer.

Additionally, the type of biopsy method was reported as a predictive factor for the upgrade of DCIS in previous studies $(5,6,11,13,14)$. CNB using 14-gauge needles can obtain a smaller amount of tissue than VAB with larger needles; therefore, patients undergoing US-guided CNB might have a higher upgrade rate than those undergoing mammography-guided VAB. However, in the present study, where most cases underwent US-guided CNB, the biopsy method was not significantly different between the DCIS and DCIS + invasion groups; therefore, it was not introduced as a predictor of DCIS upgrade.

In the present study, a higher nuclear grade of biopsy 


\begin{tabular}{|c|c|c|c|}
\hline & $\operatorname{DCIS}(n=119)$ & DCIS with invasion $(n=46)$ & P-value \\
\hline \multicolumn{4}{|l|}{ Age $(y)$} \\
\hline Mean $\pm S D$ & $54.0 \pm 10.5$ & $49.3 \pm 8.8$ & $0.008^{\mathrm{a}}$ \\
\hline Median(range) & $52.5(26.0-82.0)$ & $49.0(28.0-69.0)$ & \\
\hline \multicolumn{4}{|l|}{ Biopsy method } \\
\hline Core needle biopsy & $96(80.7)$ & $42(91.3)$ & 0.098 \\
\hline Vacuum-assisted biopsy & $23(19.3)$ & $4(8.7)$ & \\
\hline \multicolumn{4}{|l|}{ Tumor grade } \\
\hline $1 \& 2$ & $55(46.2)$ & $14(30.4)$ & 0.065 \\
\hline 3 & $64(53.8)$ & $32(69.6)$ & \\
\hline \multicolumn{4}{|l|}{$\mathbf{S U V}_{\max }$} \\
\hline Mean \pm SD & $1.9 \pm 1.1$ & $3.2 \pm 2.5$ & $<0.001^{\mathrm{a}}$ \\
\hline Median (range) & $1.6(0.7-5.6)$ & $2.5(0.7-13.5)$ & \\
\hline$<1.9$ (cutoff point) & $67(56.3)$ & $10(21.7)$ & $<0.001^{\mathrm{a}}$ \\
\hline$\geq 1.9$ (cutoff point) & $52(43.7)$ & $36(78.3)$ & \\
\hline \multicolumn{4}{|l|}{ Visual analysis of FDG-PET/CT } \\
\hline Negative & $39(32.8)$ & $4(8.7)$ & $0.002^{\mathrm{a}}$ \\
\hline Positive & $80(67.2)$ & $42(91.3)$ & \\
\hline \multicolumn{4}{|l|}{ FDG uptake of axillary LNs } \\
\hline Negative & $112(94.1)$ & $42(91.3)$ & 0.502 \\
\hline Positive & $7(5.9)$ & $4(8.7)$ & \\
\hline \multicolumn{4}{|l|}{ Pathological size $(\mathrm{DCIS})(\mathrm{cm})$} \\
\hline Mean \pm SD & $2.8 \pm 2.3$ & $4.1 \pm 2.3$ & $<0.001^{\mathrm{a}}$ \\
\hline Median(range) & $2.0(0.2-11.3)$ & $3.9(0.5-10.0)$ & \\
\hline \multicolumn{4}{|l|}{ LN metastasis in pathology } \\
\hline Negative & $118(99.2)$ & $44(95.7)$ & 0.188 \\
\hline Positive & $1(0.8)$ & $2(4.3)$ & \\
\hline \multicolumn{4}{|l|}{ ER } \\
\hline Negative & $42(35.3)$ & $15(32.6)$ & 0.745 \\
\hline Positive & $77(64.7)$ & $31(67.4)$ & \\
\hline \multicolumn{4}{|l|}{ PR } \\
\hline Negative & $49(41.2)$ & $17(37.0)$ & 0.620 \\
\hline Positive & $70(58.8)$ & $29(63.0)$ & \\
\hline \multicolumn{4}{|l|}{ HER-2 } \\
\hline Negative & $63(52.9)$ & $21(45.7)$ & 0.401 \\
\hline Positive & $56(47.1)$ & $25(54.3)$ & \\
\hline \multicolumn{4}{|l|}{ Ki-67 } \\
\hline$<14 \%$ & $62(52.1)$ & $18(39.1)$ & 0.135 \\
\hline$\geq 14 \%$ & $57(47.9)$ & $28(60.9)$ & \\
\hline \multicolumn{4}{|l|}{ EGFR } \\
\hline Negative & $86(72.3)$ & $33(71.7)$ & 0.946 \\
\hline Positive & $33(27.7)$ & $13(28.3)$ & \\
\hline
\end{tabular}

Abbreviations: DCIS, ductal carcinoma in situ; EGFR, epidermal growth factor receptor; ER, estrogen receptor; FDG-PET/CT, ${ }^{18}$ F-fluorodeoxyglucose-positron emission tomography/computed tomography; HER-2, human epidermal growth factor receptor type 2; LN, lymph node; PR, progesterone receptor; SD, standard deviation; SUV max, maximum standardized uptake value.

${ }^{a}$ P-value shows a significant difference between DCIS and IDC, based on Chi-square or Fisher's exact test and independent $t$-test or Mann-Whitney U test.

specimens was observed in the DCIS + invasion group as compared to the pure DCIS group (69.6\% vs. $53.8 \%$ ), and the difference was not statistically significant, but $P$ value was close to $<0.05(\mathrm{P}=0.065)$. However, nuclear grade was not a significant factor associated with DCIS upgrades. Gener- ally, there are contradictory results regarding the role of tumor grade. The tumor grade in biopsy $(5,12)$ and the final pathology $(2,7,11,12)$ was recognized as a significant independent predictor in some previous studies, but not in some others $(9,13,23)$. 


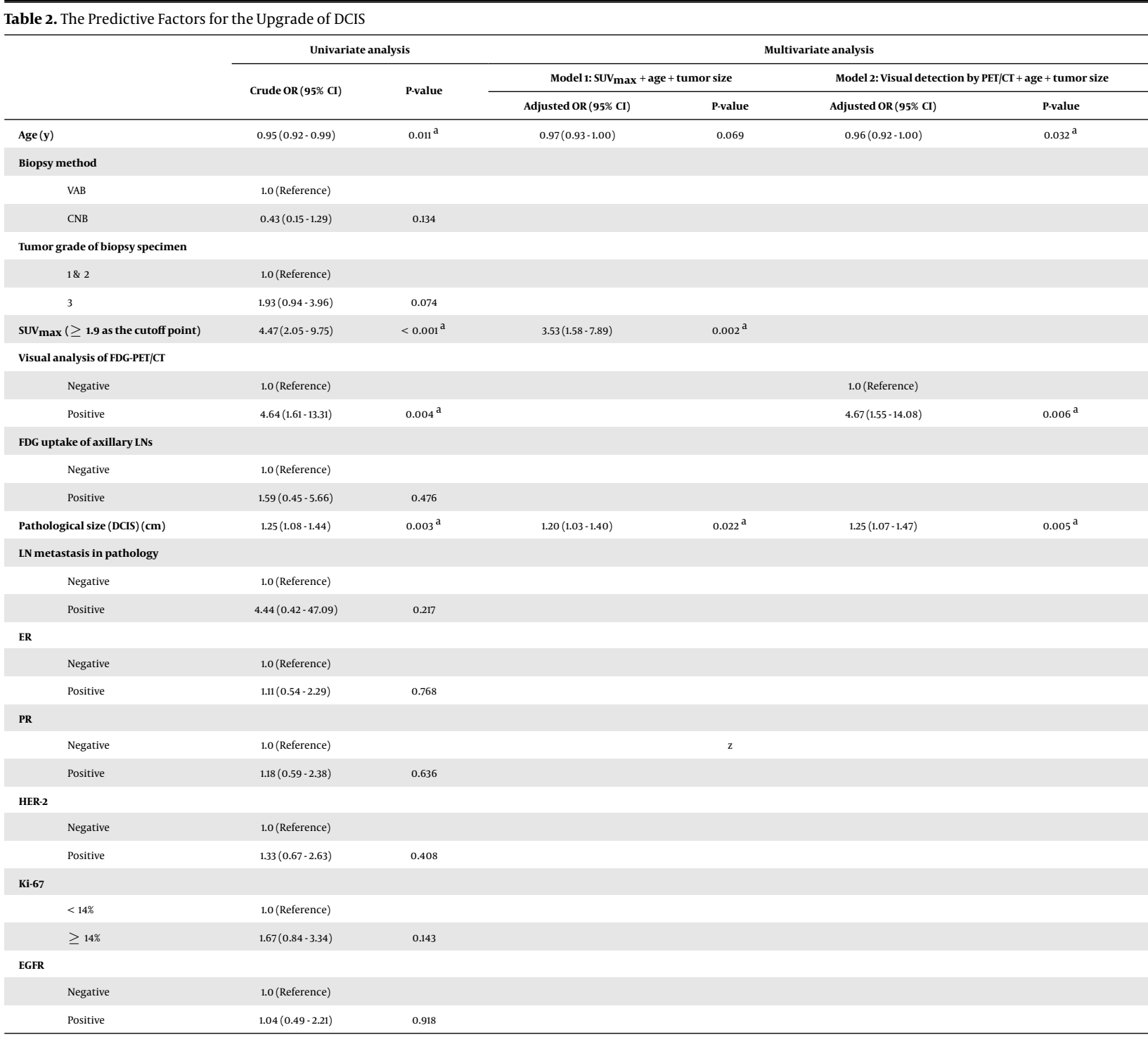

Abbreviations: CI, confidence interval; CNB, core needle biopsy; DCIS, ductal carcinoma in situ; EGFR, epidermal growth factor receptor; ER, estrogen receptor; FDG-PET/CT, ${ }^{18} \mathrm{~F}$-fluorodeoxyglucose-positron emission tomography/computed tomography; HER-2, human epidermal growth factor receptor type 2; LN, lymph node; OR, odds ratio; PR, progesterone receptor; SUV max, maximum standardized uptake value; VAB, vacuum-assisted biopsy. ${ }^{\mathrm{a}}$ Statistically significant.

Table 3. Comparison of the Diagnostic Performance of Visual and Semi-quantitative Analyses of FDG-PET/CT scan

\begin{tabular}{|c|c|c|c|c|c|}
\hline & Sensitivity (95\% CI) & Specificity (95\% CI) & $\operatorname{PPV}(95 \% \mathrm{CI})$ & $\operatorname{NPV}(95 \% \mathrm{CI})$ & $\operatorname{Accuracy}(95 \% \mathrm{CI})$ \\
\hline $\begin{array}{l}\text { Semi-quantitative analysis with } \\
\operatorname{SUV}_{\max }(\geq 1.9, \text { cutoff point })\end{array}$ & $0.78(0.64-0.89)$ & $0.56(0.47-0.65)$ & $0.41(0.31-0.52)$ & $0.87(0.77-0.94)$ & $0.62(0.55-0.70)$ \\
\hline Visual analysis & $0.91(0.79-0.98)$ & $0.33(0.24-0.42)$ & $0.34(0.26-0.44)$ & $0.91(0.78-0.97)$ & $0.49(0.41-0.57)$ \\
\hline P-value (visual vs. semi-quantitative) & $0.014^{\mathrm{a}}$ & $<0.001^{\mathrm{a}}$ & $0.012^{\mathrm{a}}$ & 0.297 & $<0.001^{\mathrm{a}}$ \\
\hline
\end{tabular}

Abbreviations: FDG-PET/CT, 18F-fluorodeoxyglucose-positron emission tomography/computed tomography; SUVmax, maximum standardized uptake value; CI, confidence interval; PPV, positive predictive value; NPV, negative predictive value.

${ }^{\text {a }}$-value for differences was determined using McNemar test (sensitivity, specificity, and accuracy) or generalized score statistic (PPV and NPV). 
In previous studies $(5-7,11,13,15)$, tumor size was reported as one of the significant factors for DCIS upgrade. In this study, the pathological tumor size was significantly larger in the DCIS group with invasion as compared to the pure DCIS group, which is consistent with previous studies $(7,9,13,15)$. The DCIS upgrade was also significantly correlated with the pathological tumor size in the present study, similar to previous research $(5,9,13)$. Moreover, in the present study, immunohistochemical markers, previously known as prognostic factors for breast cancer, were not correlated with upgrade to invasive cancer from DCIS in biopsy. Also, there are some studies on the relationship between DCIS upgrade and immunohistochemical markers $(2,7,14,15)$; however, their results are inconsistent, and consensus has not been reached. In this study, we did not find any correlation between DCIS upgrade and immunohistochemical markers, which is in line with a previous study (22).

The present findings revealed that visual and semiquantitative analyses of FDG-PET/CT could predict DCIS upgrade preoperatively, independent of other prognostic clinicopathological factors. The $\mathrm{SUV}_{\max }$ can be an objective indicator and a practical tool to predict the upgrade of DCIS. Comparison of diagnostic performance between the semi-quantitative and visual analyses showed that the visual analysis was more sensitive for detecting the invasion of DCIS; however, the overall accuracy of the semiquantitative analysis was significantly higher than that of the visual analysis.

The main limitation of this study was its retrospective design, which could induce selection bias. Second, it was difficult in some cases to determine the correlation between the exact location of a biopsy-proven malignancy on conventional images, FDG uptake on PET/CT scan, and pathological lesion after surgery; however, we tried to find the lesion at least in the same quadrant in all cases. Third, because biopsy was performed before FDG-PET/CT scan for all patients, it could affect the PET/CT results. Finally, the menstrual cycle and menopause status, which could affect the diagnosis of FDG-PET/CT, were not considered in this study. Therefore, a well-designed prospective study is needed for further evaluation of the efficacy of FDG-PET/CT scan in detecting the invasive components of DCIS compared to other imaging modalities and pathological parameters.

In conclusion, this study demonstrated that both semiquantitative and visual analyses of FDG uptake could preoperatively predict the risk of upgrade to invasive cancer, independent of other clinicopathological characteristics.

\section{Acknowledgments}

The statistical consultation in this study was granted by Korea Health Technology R\&D Project of Korea Health Industry Development Institute (KHIDI), funded by the Ministry of Health and Welfare, Republic of Korea (grant number: HI14C1062).

\section{Footnotes}

Authors' Contributions: B.J.K. conceived and designed the study; B.J.K and N.Y.J. collected the clinical data, interpreted the data, and contributed to the statistical analysis and drafting of the manuscript; S.H.K. and I.R.Y. participated in designing the study and drafting the manuscript; Y.S.L. re-analyzed the clinical and statistical data and revised the manuscript; and W.J.Y. critically revised the manuscript. All authors read and approved the final manuscript.

Conflict of Interests: The authors declare that they have no competing interests.

Ethical Approval: No ethical approval was required for this retrospective study, because it included the collection and analysis of preexisting clinical data.

Funding/Support: The authors declare that they did not receive any funding for this study.

\section{References}

1. Fujioka T, Kubota K, Toriihara A, Machida Y, Okazawa K, Nakagawa $\mathrm{T}$, et al. Tumor characteristics of ductal carcinoma in situ of breast visualized on [F-18] fluorodeoxyglucose-positron emission tomography/computed tomography: Results from a retrospective study. World J Radiol. 2016;8(8):743-9. doi: 10.4329/wjr.v8.i8.743. [PubMed: 27648168]. [PubMed Central: PMC5002505].

2. Seo YY, Yoo IR, Park SY, Oh JK, Kim SH, Sohn HS. Ductal carcinoma in situ and ductal carcinoma in situ with microinvasion: correlation of FDG uptake with histological and biological prognostic factors. Breast Cancer. 2017;24(3):353-61. doi: 10.1007/s12282-016-0710-7. [PubMed: 27312540].

3. Parikh U, Chhor CM, Mercado CL. Ductal carcinoma in situ: The whole truth.AJRAm JRoentgenol.2018;210(2):246-55. doi:10.2214/AJR.17.18778. [PubMed: 29045181].

4. Shigematsu H, Kadoya T, Masumoto N, Matsuura K, Emi A, Kajitani $\mathrm{K}$, et al. Role of FDG-PET/CT in prediction of underestimation of invasive breast cancer in cases of ductal carcinoma in situ diagnosed at needle biopsy. Clin Breast Cancer. 2014;14(5):358-64. doi: 10.1016/j.clbc.2014.04.006. [PubMed: 24962555].

5. Brennan ME, Turner RM, Ciatto S, Marinovich ML, French JR, Macaskill $P$, et al. Ductal carcinoma in situ at core-needle biopsy: metaanalysis of underestimation and predictors of invasive breast cancer. Radiology. 2011;260(1):119-28. doi: 10.1148/radiol.11102368. [PubMed: 21493791].

6. Houssami N, Ciatto S, Ellis I, Ambrogetti D. Underestimation of malignancy of breast core-needle biopsy: concepts and precise overall and category-specific estimates. Cancer. 2007;109(3):487-95. doi: 10.1002/cncr.22435. [PubMed: 17186530]. 
7. Chavez de Paz Villanueva C, Bonev V, Senthil M, Solomon N, Reeves ME, Garberoglio CA, et al. Factors associated with underestimation of invasive cancer in patients with ductal carcinoma in situ: Precautions for active surveillance. JAMA Surg. 2017;152(11):1007-14. doi: 10.1001/jamasurg.2017.2181. [PubMed: 28700803]. [PubMed Central: PMC5710412].

8. Ryser MD, Worni M, Turner EL, Marks JR, Durrett R, Hwang ES. Outcomes of active surveillance for ductal carcinoma in situ: A computational risk analysis. J Natl Cancer Inst. 2016;108(5). doi: 10.1093/jnci/djv372. [PubMed: 26683405]. [PubMed Central: PMC4849804].

9. Huo L, Sneige N, Hunt KK, Albarracin CT, Lopez A, Resetkova E. Predictors of invasion in patients with core-needle biopsy-diagnosed ductal carcinoma in situ and recommendations for a selective approach to sentinel lymph node biopsy in ductal carcinoma in situ. Cancer. 2006;107(8):1760-8. doi: 10.1002/cncr.22216. [PubMed: 16977650].

10. Kerlikowske K, Molinaro AM, Gauthier ML, Berman HK, Waldman F, Bennington J, et al. Biomarker expression and risk of subsequent tumors after initial ductal carcinoma in situ diagnosis. J Natl Cancer Inst. 2010;102(9):627-37. doi: 10.1093/jnci/djq101. [PubMed: 20427430]. [PubMed Central: PMC2864293].

11. Yen TW, Hunt KK, Ross MI, Mirza NQ, Babiera GV, Meric-Bernstam F, et al. Predictors of invasive breast cancer in patients with an initial diagnosis of ductal carcinoma in situ: a guide to selective use of sentinel lymph node biopsy in management of ductal carcinoma in situ. J Am Coll Surg. 2005;200(4):516-26. doi: 10.1016/j.jamcollsurg.2004.11.012. [PubMed: 15804465].

12. Bagnall MJ, Evans AJ, Wilson AR, Pinder SE, Denley H, Geraghty JG, et al. Predicting invasion in mammographically detected microcalcification. Clin Radiol. 2001;56(10):828-32. doi:10.1053/crad.2001.0779. [PubMed: 11895299].

13. Dillon MF, McDermott EW, Quinn CM, O'Doherty A, O'Higgins N, Hill AD. Predictors of invasive disease in breast cancer when core biopsy demonstrates DCIS only. J Surg Oncol. 2006;93(7):559-63. doi: 10.1002/jso.20445. [PubMed: 16705731].

14. Lee CW, Wu HK, Lai HW, Wu WP, Chen ST, Chen DR, et al. Preoperative clinicopathologic factors and breast magnetic resonance imaging features can predict ductal carcinoma in situ with invasive components. Eur J Radiol. 2016;85(4):780-9. doi: 10.1016/j.ejrad.2015.12.027. [PubMed: 26971424].

15. Yu KD, Wu LM, Liu GY, Wu J, Di GH, Shen ZZ, et al. Different distribution of breast cancer subtypes in breast ductal carcinoma in situ (DCIS), DCIS with microinvasion, and DCIS with invasion component. Ann Surg Oncol. 2011;18(5):1342-8. doi: 10.1245/s10434-010-1407-3. [PubMed: 21042943].

16. Ekmekcioglu O, Aliyev A, Yilmaz S, Arslan E, Kaya R, Kocael P, et al. Correlation of $18 \mathrm{~F}-$ fluorodeoxyglucose uptake with histopathological prognostic factors in breast carcinoma. Nucl Med Commun 2013;34(11):1055-67. doi: 10.1097/MNM.ob013e3283658369. [PubMed: 24025919].

17. Groheux D, Giacchetti S, Moretti JL, Porcher R, Espie M, Lehmann-Che J, et al. Correlation of high 18F-FDG uptake to clinical, pathological and biological prognostic factors in breast cancer. Eur J Nucl Med Mol Imaging. 2011;38(3):426-35. doi: 10.1007/s00259-010-1640-9. [PubMed: 21057787].

18. Koolen BB, Vrancken Peeters MJ, Wesseling J, Lips EH, Vogel WV, Aukema TS, et al. Association of primary tumour FDG uptake with clinical, histopathological and molecular characteristics in breast cancer patients scheduled for neoadjuvant chemotherapy. Eur J Nucl Med Mol Imaging. 2012;39(12):1830-8. doi: 10.1007/s00259-012-2211-Z. [PubMed: 22895862]

19. Sanli Y, Kuyumcu S, Ozkan ZG, Isik G, Karanlik H, Guzelbey B, et al. Increased FDG uptake in breast cancer is associated with prognostic factors. Ann Nucl Med. 2012;26(4):345-50. doi: 10.1007/s12149-012-0579-2. [PubMed: 22359222].

20. Avril N, Rose CA, Schelling M, Dose J, Kuhn W, Bense S, et al. Breast imaging with positron emission tomography and fluorine-18 fluorodeoxyglucose: use and limitations. J Clin Oncol. 2000;18(20):3495502. doi: 10.1200/JCO.2000.18.20.3495. [PubMed: 11032590].

21. Avril N, Menzel M, Dose J, Schelling M, Weber W, Janicke F, et al. Glucose metabolism of breast cancer assessed by 18F-FDG PET: histologic and immunohistochemical tissue analysis. J Nucl Med. 2001;42(1):916. [PubMed: 11197987].

22. Fujii T, Yanai K, Tokuda S, Nakazawa Y, Kurozumi S, Obayashi S, et al Clinicopathological features of ductal carcinoma in situ from (18)FFDG-PET findings. Anticancer Res. 2017;37(9):5053-6. doi: 10.21873/anticanres.11921. [PubMed: 28870933].

23. Kim S, Lee S, Kim S, Lee S, Yum $H$. The usefulness of fluorodeoxyglucose-PET/CT for preoperative evaluation of ductal carcinoma in situ. Ann Surg Treat Res. 2018;94(2):63-8. doi: 10.4174/astr.2018.94.2.63. [PubMed: 29441334]. [PubMed Central: PMC5801329]. 